\title{
POWER-CENTRAL POLYNOMIALS ON MATRICES
}

\author{
ALEXEY KANEL-BELOV, SERGEY MALEV, LOUIS ROWEN
}

\begin{abstract}
Any multilinear non-central polynomial $p$ (in several noncommuting variables) takes on values of degree $n$ in the matrix algebra $M_{n}(F)$ over an infinite field $F$. The polynomial $p$ is called $\nu$-central for $M_{n}(F)$ if $p^{\nu}$ takes on only scalar values, with $k$ minimal such. Multilinear $\nu$-central polynomials do not exist for any $\nu$ with $n>3$, thereby answering a question of Drensky. Saltman proved that an arbitrary polynomial $p$ cannot be $\nu$-central for $M_{n}(F)$ for $n$ odd unless $n$ is prime; we show for $n$ even, that $\nu$ must be 2 .
\end{abstract}

\section{INTRODUCTION}

For any polynomial $p \in K\left\langle x_{1}, \ldots, x_{m}\right\rangle$, the image of $p$ (in $R$ ), denoted $\operatorname{Im} p$, is defined as

$$
\left\{r \in R: \text { there exist } a_{1}, \ldots, a_{m} \in R \text { such that } p\left(a_{1}, \ldots, a_{m}\right)=r\right\} .
$$

Remark 1. Im $p$ is invariant under conjugation, since

$$
a p\left(x_{1}, \ldots, x_{m}\right) a^{-1}=p\left(a x_{1} a^{-1}, a x_{2} a^{-1}, \ldots, a_{m} a^{-1}\right) \in \operatorname{Im} p,
$$

for any nonsingular $a \in M_{n}(K)$.

This note is an outgrowth of [BMR1] and BMR2, in which we considered the question, reputedly raised by Kaplansky, of $\operatorname{Im} p$ on $n \times n$ matrices. (We take $n$ to be given throughout this note, as is our base field $K$.)

Specifically, the following conjecture is attributed to Kaplansky:

Conjecture 1. If $p$ is a multilinear polynomial evaluated on the matrix ring $M_{n}(K)$, then $\operatorname{Im} p$ is either $\{0\}, K$ (viewed as $K$ the set of scalar matrices), $\operatorname{sl}_{n}(K)$, or $M_{n}(K)$.

Here is one celebrated example.

Definition 1. A polynomial $p$ is central (with respect to $M_{n}(K)$ ) if $p$ takes on only scalar values, but does not vanish identically.

The existence of multilinear central polynomials for $n \times n$ matrices was proved by Formanek [F1] and Razmyslov [Ra1]. Furthermore, it has long been known that $\left[x_{1}, x_{2}\right]^{2}$ is central for $2 \times 2$ matrices, and one of the possibilities arising for $3 \times 3$ matrices is a polynomial whose cube is central, thereby motivating the following definition:

Definition 2. A polynomial $p$ is $\nu$-central if $p^{\nu}$ is central, for $\nu \geq 1$ minimal such. The polynomial $p$ is power-central if $p$ is $\nu$-central, for some $\nu>1$.

This research was supported by the Israel Science Foundation (grant no. 1207/12).

The second named author was supported by an Israeli Ministry of Immigrant Absorbtion scholarship. 
Our objective here is to examine the existence of $\nu$-central polynomials. For $n=\nu$ prime, as explained in Row, Theorem 3.2.20], this is equivalent to Amitsur's generic division algebra being cyclic, one of the major open questions in the theory of division algebras, and we have nothing more to say about this case, but we can solve the other cases.

Remark 2. A homogeneous 3-central polynomial for $n=3$ was constructed in Row, Theorem 3.2.21], and a homogeneous 2-central polynomial for $n=4$ was constructed in Row, Proposition 3.2.24].

Using the structure theory of division algebras, Saltman $[\underline{\mathrm{S}}]$ proved that in characteristic $0, \nu$-central polynomials do not exist for odd $\nu>1$ unless $n$ is prime. This led Drensky to ask what happens for $\nu=2$, noting the result of Remark 2 We consider both the homogeneous case and the more restrictive multilinear case.

Our main result is:

Theorem 5, Assume $n \geq 4$. For Char $(K)$ arbitrary, there are no multilinear power central polynomials. Any multilinear polynomial is either PI, or central, or its image in $M_{n}(K)$ is at least $\left(n^{2}-n+2\right)$-dimensional.

This implies easily that multilinear $\nu$-central polynomials do not exist unless $\nu=n$. One can conclude via structure theory that:

(Theorem 1) 4-central polynomials do not exist, and (Theorem 4) multilinear 2-central polynomials do not exist unless $n=2$.

Then, by examining dimensions of images, we conclude in Theorem 5 for arbitrary $\nu$, that multilinear $\nu$-central polynomials do not exist whenever $n \geq 4$.

\section{Considerations ARISING From Division ALGEBRAS}

These questions (for $\nu$ a power of 2 ) have easy answers for homogeneous polynomials. Much of the material in this section is standard (although we do not have a specific reference), obtained from well-known facts about division algebras. We say an element $d$ of a division algebra $D$ with center $F$ is $\nu$-central if $d^{\nu} \in F$ but $d^{\ell} \notin F$ for any $\ell$ dividing $\nu, \nu$ minimal such. We say a subspace $V$ is $\nu$-central if every element is $\ell$-central for some $\ell$ dividing $\nu$.

A major tool is Amitsur's Theorem [Row, Theorem 3.2.6, p. 176], that the algebra of generic $n \times n$ matrices (generated by matrices $Y_{k}=\left(\xi_{i, j}^{(k)}\right)$ whose entries $\left\{\xi_{i, j}^{(k)}, 1 \leq i, j \leq n\right\}$ are commuting indeterminates) is a non-commutative domain UD whose ring of fractions with respect to the center is a division algebra which we denote as $\widetilde{\mathrm{UD}}$ of dimension $n^{2}$ over its center $\left.F_{1}:=\operatorname{Cent}(\widetilde{\mathrm{UD}})\right)$.

We shall need a general fact about polynomial evaluations.

Lemma 1. For any polynomial $p\left(x_{1}, \ldots, x_{m}\right)$ which has an evaluation of degree $n$ on $M_{n}(F)$, there is an index $i, 1 \leq i \leq m$, and matrices $a_{1}, a_{2}, \ldots, a_{m}, a_{i}^{\prime}$ such that the evaluations $p\left(a_{1}, \ldots, a_{i-1}, a_{i}, a_{i+1}, \ldots, a_{m}\right)$ and $p\left(a_{1}, \ldots, a_{i-1}, a_{i}^{\prime}, a_{i+1}, \ldots, a_{m}\right)$ do not commute.

Proof. We go back and forth to generic matrices and $\widetilde{\mathrm{UD}}$. First of all, for all generic matrices $Y_{1}, \ldots, Y_{m}, Y_{1}^{\prime}, \ldots, Y_{m}^{\prime}$, and each $i$, clearly $p\left(Y_{1}, \ldots, Y_{i}, Y_{i+1}^{\prime}, \ldots, Y_{m}^{\prime}\right)$ has degree $n$ over $F_{1}$, and thus has distinct eigenvalues, from which it follows at once that $p\left(Y_{1}, \ldots, Y_{m}\right)$ and $p\left(Y_{1}^{\prime}, \ldots, Y_{m}^{\prime}\right)$ do not commute (since one could diagonalize $p\left(Y_{1}, \ldots, Y_{m}\right)$ while $p\left(Y_{1}^{\prime}, \ldots, Y_{m}^{\prime}\right)$ remains non-diagonal $)$. 
But, for each $i, F_{1}\left(p\left(Y_{1}, \ldots, Y_{i}, Y_{i+1}^{\prime}, \ldots, Y_{m}^{\prime}\right)\right)$ has dimension $n$ over $F_{1}$ and thus is a maximal subfield of $\widetilde{\mathrm{UD}}$. It follows that $p\left(Y_{1}, \ldots, Y_{i}, Y_{i+1}^{\prime}, \ldots, Y_{m}^{\prime}\right)$ and $p\left(Y_{1}, \ldots, Y_{j-1}, Y_{j}^{\prime}, \ldots, Y_{m}^{\prime}\right)$ commute iff

$$
F_{1}\left(p\left(Y_{1}, \ldots, Y_{i}, Y_{i+1}^{\prime}, \ldots, Y_{m}^{\prime}\right)\right)=F_{1}\left(p\left(Y_{1}, \ldots, Y_{j-1}, Y_{j}^{\prime}, \ldots, Y_{m}^{\prime}\right)\right) .
$$

In particular, $F_{1}\left(p\left(Y_{1}, \ldots, Y_{m}\right)\right) \neq F_{1}\left(p\left(Y_{1}^{\prime}, \ldots, Y_{m}^{\prime}\right)\right)$, implying

$$
F_{1}\left(p\left(Y_{1}, \ldots, Y_{i}, Y_{i+1}^{\prime}, \ldots, Y_{m}^{\prime}\right)\right) \neq F_{1}\left(p\left(Y_{1}, \ldots, Y_{i-1}, Y_{i}^{\prime}, \ldots, Y_{m}^{\prime}\right)\right)
$$

for some $i$, and thus $p\left(Y_{1}, \ldots, Y_{i}, Y_{i+1}^{\prime}, \ldots, Y_{m}^{\prime}\right)$ and $p\left(Y_{1}, \ldots, Y_{i-1}, Y_{i}^{\prime} \ldots, Y_{m}^{\prime}\right)$ do not commute. In other words,

$$
\left[p\left(Y_{1}, \ldots, Y_{i}, Y_{i+1}^{\prime}, \ldots, Y_{m}^{\prime}\right), p\left(Y_{1}, \ldots, Y_{i-1}, Y_{i}^{\prime} \ldots, Y_{m}^{\prime}\right)\right] \neq 0
$$

implying there is a specialization

$$
Y_{1} \mapsto a_{1}, \ldots, Y_{i} \mapsto a_{i}, Y_{i}^{\prime} \mapsto a_{i}^{\prime}, Y_{i+1}^{\prime} \mapsto a_{i+1}, \ldots, Y_{m}^{\prime} \mapsto a_{m}
$$

yielding $\left[p\left(a_{1}, \ldots, a_{i-1}, a_{i}, a_{i+1}, \ldots, a_{m}\right), p\left(a_{1}, \ldots, a_{i-1}, a_{i}^{\prime}, a_{i+1}, \ldots, a_{m}\right)\right] \neq 0$.

We return to power-central polynomials.

Remark 3. The existence of a $\nu$-central polynomial is equivalent to UD containing an element whose $\nu$-power is central, with $\nu$ minimal such. On the other hand, any such element can be specialized to an arbitrary division algebra of dimension $n^{2}$ over its center. Thus, to prove the non-existence of a $\nu$-central polynomial, it suffices for suitable $\ell$ dividing $\nu$ to construct a division algebra with center $F \supseteq K$ having the property that if $d^{\nu} \in F$ then $d^{\ell} \in F$.

Example 1. Suppose $K$ has characteristic $\neq 2$, and $n=2^{t-1} q$ where $q$ is odd, and construct $D$ to be a tensor product of "generic" symbols $\left(\lambda_{u}^{n_{u}}, \mu_{u}^{n_{u}}\right)$ as in Row, Example 7.1.28 and Theorem 7.1.29], where $n_{1}=n_{2}=\cdots=n_{t-1}=2$ and $n_{t}=q$. In other words, $D$ is the algebra of central fractions of the skew polynomial $\operatorname{ring} R:=$ $K(\rho)\left[\lambda_{u} \mu_{u}: 1 \leq u \leq t\right]$ where $\rho$ is a primitive $q$ root of 1 and the indeterminates commute except for $\lambda_{u} \mu_{u}=-\mu_{u} \lambda_{u}$ for $1 \leq u \leq t-1$ and $\lambda_{t} \mu_{t}=\rho \mu_{t} \lambda_{t}$. We write a typical element of $R$ as $\sum \alpha_{\mathbf{i}, \mathbf{j}} \lambda^{\mathbf{i}} \mu^{\mathbf{j}}$, where $\lambda^{\mathbf{i}}$ denotes $\prod_{u=1}^{t} \lambda_{u}^{i_{u}}$. There is a natural grade given by the lexicographic order on the exponents of the monomials, and it is easy to see that if $d^{\nu} \in F$ then the leading term $\hat{d}^{\nu} \in F$.

In particular, for $\nu=2$, if $d^{2} \in F$ then $\hat{d}$ must have the form $\alpha \lambda^{\mathbf{i}} \mu^{\mathbf{j}}$ where $i_{t}=j_{t}=0$. On the other hand, we claim that if $d^{2} \in F$ and $\hat{d} \in F$, then $d \in F$. Indeed, taking $d^{\prime}$ to be the next leading term in $d$, we have

$$
d^{2}=\left(\hat{d}+d^{\prime}\right)^{2}=\hat{d}^{2}+2 \hat{d} d^{\prime}+\cdots,
$$

implying $d^{\prime} \in F$, and continuing, we conclude $d \in F$, as desired.

It follows that if $d$ is 2-central then $\hat{d}$ is 2-central.

Now we claim that $D$ does not have 4-central elements. Indeed, if $d$ is 4-central then $d^{2}$ is 2-central, implying $\hat{d}^{2}$ is 2-central, and thus $\hat{d}$ is 4-central, implying $\hat{d}$ must have the form $\alpha \lambda^{\mathbf{i}} \mu^{\mathbf{j}}$ where $i_{t}=j_{t}=0$; we conclude that $\hat{d}$ is is 2-central, implying $\hat{d}^{2} \in F$, and thus $d^{2} \in F$ by the claim.

Theorem 1. There do not exist 4-central polynomials.

Proof. Combine Remark 3 with Example 1 .

This leaves us with 2-central polynomials. 
Proposition 1. There exist homogeneous 2-central polynomials with respect to $M_{n}(F)$ if $n=2 q$ or $n=4 q$ for $q$ odd.

Proof. $\widetilde{\mathrm{UD}}$ is a tensor product of a division algebra $D_{1}$ of degree 2 or 4 and a division algebra of degree $q$, and we observed earlier that $D_{1}$ has a 2-central element.

The situation for $8 \mid n$ remains open, and is equivalent to another important question in division algebras about the existence of square-central elements. The following observation might be relevant, although we do not use it further.

Lemma 2. Suppose $R=M_{2}(D)$ with $D$ an F-central division algebra. The 2central elements of $R$ have the form

$$
\left(\begin{array}{cc}
a & b \\
-b^{-1}\left(a^{2}-\alpha\right) & -b^{-1} a b
\end{array}\right): \alpha \in F, a, b \in D .
$$

Proof. Consider any square-central matrix $A=\left(\begin{array}{ll}a & b \\ c & d\end{array}\right)$. Then

$$
A^{2}=\left(\begin{array}{ll}
a^{2}+b c & a b+b d \\
c a+d c & c b+d^{2}
\end{array}\right)
$$

so we must have $\alpha \in F$ such that:

(i) $a^{2}+b c=\alpha$;

(ii) $c b+d^{2}=\alpha$;

(iii) $a b+b d=0$;

(iv) $c a+d c=0$.

(i) implies $c=-b^{-1}\left(a^{2}-\alpha\right)$. Then (ii) implies

$$
\left.d^{2}=\alpha+b^{-1}\left(a^{2}-\alpha\right)\right) b=b^{-1} a^{2} b=\left(b^{-1} a b\right)^{2},
$$

so $d= \pm b^{-1} a b$. But only $d=-b^{-1} a b$ works. Thus the matrix is in the form of (1), and this is indeed square-central.

Note that this enables one to construct "generic" 2-central elements, by choosing $a$ and $b$ and $\alpha$ arbitrarily, but we do not know if they occur as elements of UD.

\section{Multilinear 2-Central Polynomials}

Having settled the issue for homogeneous polynomials except for $n=8 q$, we turn to multilinear polynomials, where the story ends differently. Although we will get a more general result, it is instructive to start with the division algebra approach.

Lemma 3. Any division algebra $D$ with a 2-central subspace $V$ of dimension 2 contains an $F$-central quaternion subalgebra. In particular, $n:=\operatorname{deg}(D)$ cannot be odd. Also, 4 does not divide $n$ if $D$ also has exponent $n$.

Proof. Take $v, v^{\prime} \in V$. Then $v^{2}, v^{\prime 2} \in F$. But also, by assumption, $v+v^{\prime}$ is also square-central, so

$$
v^{2}+v v^{\prime}+v^{\prime} v+v^{\prime 2}=\left(v+v^{\prime}\right)^{2} \in F,
$$

implying $v^{\prime} v=-v v^{\prime}+\alpha$ for some $\alpha \in F$. But then $F+F v+F v^{\prime}+F v v^{\prime}$ is a central $F$-subalgebra of $D$ and has dimension at least 3 , but has elements of degree 2 , so has dimension 4 . 
The last assertion follows easily from the theory of finite dimensional division algebras. If $D$ has a quaternion division algebra then 2 must divide $n$ and the exponent of $D$ is the least common multiple of 2 and $\frac{n}{2}$.

Lemma 4. If $p\left(x_{1}, \ldots, x_{m}\right)$ is a 2-central polynomial for $n \times n$ matrices, linear in $x_{1}$, and there are non-commuting values $p\left(a_{1}, \ldots, a_{m}\right)$ and $p\left(a_{1}^{\prime}, \ldots, a_{m}\right)$ for matrices $a_{1}, a_{1}^{\prime}, a_{2}, \ldots, a_{m}$, then the generic division algebra $\widetilde{\mathrm{UD}}$ of degree $n$ has a $F_{1}$-central quaternion subalgebra. In particular, $n$ cannot be odd, and 4 does not divide $n$.

Proof. Let

$$
w=p\left(Y_{1}, \ldots, Y_{m}\right), \quad w^{\prime}=p\left(Y_{1}^{\prime}, Y_{2} \ldots, Y_{m}\right),
$$

where the $Y_{i}$ and $Y_{1}^{\prime}$ are generic matrices. Then $w^{2}, w^{\prime 2} \in F_{1}$. But also, by definition, $w+w^{\prime}=p\left(Y_{1}+Y_{1}^{\prime}, \ldots, Y_{m}\right)$ is also 2-central, so

$$
w^{2}+w w^{\prime}+w^{\prime} w+{w^{\prime}}^{2}=\left(w+w^{\prime}\right)^{2} \in F_{1},
$$

implying $w^{\prime} w=-w w^{\prime}+\alpha$ for some $\alpha \in F_{1}$. But then $F_{1}+F_{1} w+F_{1} w^{\prime}+F_{1} w w^{\prime}$ is a central $F_{1}$-subalgebra of $\widetilde{\mathrm{UD}}$ and has dimension 4 .

The last assertion follows since the generic division algebra $\widetilde{\mathrm{UD}}$ of degree $n$ has exponent $n$, whereas if $\widetilde{\mathrm{UD}}$ has a central quaternion division subalgebra, then 2 must divide $n$ and the exponent of $\widetilde{\mathrm{UD}}$ is the least common multiple of 2 and $\frac{n}{2}$.

Theorem 2. If $p\left(x_{1}, \ldots, x_{m}\right)$ is a multilinear 2-central polynomial for $n \times n m a$ trices, then the generic division algebra of degree $n$ has a quaternion part. In particular, $n$ cannot be odd, and 4 does not divide $n$.

Proof. In view of Lemma 1, two consecutive terms of the chain

$$
p\left(a_{1}, \ldots, a_{m}\right), p\left(a_{1}^{\prime}, a_{2} \ldots, a_{m}\right), \cdots, p\left(a_{1}^{\prime}, \ldots, a_{m}^{\prime}\right)
$$

do not commute, so conclude with Lemma 4 .

This conclusion is the opposite of Proposition 1, when $n=4 q$ for $q$ odd.

For $n>2$, we also have an easy consequence of the theory of division algebras.

Lemma 5. If a polynomial $p$ is $\nu$-central for $M_{n}(K)$ for $\nu>1$, then $\nu$ cannot be relatively prime to $n$.

Proof. We can view $p$ as an element of the generic division algebra $\widetilde{\mathrm{UD}}$ of degree $n$, and we adjoin an $\nu$-root of 1 to $K$ if necessary. Then $p$ generates a subfield of $\widetilde{\mathrm{UD}}$, of dimension dividing $\nu$. Hence the dimension is 1 ; i.e., $\nu=1$.

\section{Multilinear polynomials evaluated on $n \times n$ Matrices}

To handle the remaining 2-central case, where $n=2 q$ for $q$ odd, we need to dig deeper into the computations. We prove a result that also applies for arbitrary $m$, for $n>3$. We need the following well-known lemma about Eulerian graphs, cf. [BMR1, Lemma 4]:

Lemma 6. If $a_{i}$ are matrix units, then $p\left(a_{1}, \ldots, a_{m}\right)$ is either 0 or a diagonal matrix, or $\alpha e_{i j}$ for some $\alpha \in K$ and $i \neq j$.

Theorem 3. Let $p\left(x_{1}, \ldots, x_{m}\right)$ be any multilinear polynomial evaluated on $n \times n$ matrices over an infinite field. Assume that $p$ is neither scalar nor PI. Then Im $p$ contains a matrix with eigenvalues $\left\{c, c \varepsilon, \ldots, c \varepsilon^{n-1}\right\}$ for some $0 \neq c \in K$. 
Proof. Define $\chi$ to be the permutation of the set of matrix units, sending the index $i \mapsto i+1$ for $1 \leq i \leq n-1$, and $n \mapsto 1$. For example, $\chi\left(e_{12}\right)=e_{23}, \chi\left(e_{57}\right)=e_{68}$. Since we substitute only matrix units into $p$, Lemma 6 shows that the image is either diagonal or a matrix unit with some coefficient. Consider the corresponding graph $\Gamma$. If the graph is an Eulerian cycle then this sum is 0, and if it is an Eulerian path from $k$ to $\ell$ then this sum equals $\ell-k$. By Lemma 6 there exist matrix units $a_{1}, \ldots, a_{m}$ with $p\left(a_{1}, \ldots, a_{m}\right)=\alpha e_{12}$ for some $\alpha \in K$. We may assume that $a_{1} \cdots a_{m}=e_{12}$. Writing $a_{\ell}=e_{i_{\ell}, j_{\ell}}$, we define $\iota\left(a_{\ell}\right)=i_{\ell}-j_{\ell}$. Thus $i_{1}=1$ and $j_{m}=2$, and $\sum \iota\left(a_{\ell}\right)=1$. Then $\chi^{k}\left(a_{\ell}\right)=e_{i_{\ell}+k, j_{\ell}+k}$ (taken modulo $n$ ), implying

$$
\iota\left(\chi^{k}\left(a_{\ell}\right)\right) \equiv\left(i_{\ell}+k\right)-\left(j_{\ell}+k\right)=i_{\ell}-j_{\ell}=\iota\left(a_{\ell}\right) \quad(\bmod n) .
$$

consider

$$
f\left(a_{1}, \ldots, a_{m}\right)=p\left(\sum_{k_{1}=1}^{m} t_{k_{1}, 1} \chi^{k_{1}}\left(a_{1}\right), \ldots, \sum_{k_{m}=1}^{m} t_{k_{m}, m} \chi^{k_{m}}\left(a_{m}\right)\right),
$$

where the $t_{k, \ell}$ are commuting indeterminates. Opening the brackets, we have $n^{m}$ terms, each of the form

$$
a^{\prime}=\chi^{k_{1}}\left(a_{\pi(1)}\right) \cdots \chi^{k_{m}}\left(a_{\pi(m)}\right)
$$

which, if nonzero, must have

$$
\iota\left(a^{\prime}\right) \equiv \sum_{\ell=1}^{m} \iota\left(\chi^{k_{\ell}} a_{\pi(\ell)}\right) \equiv \sum_{\ell=1}^{m} \iota\left(a_{\pi(\ell)}\right) \equiv 1 \quad(\bmod n),
$$

implying $a^{\prime}$ is a matrix of the form $c e_{i, i+1}$ or $c e_{n, 1}$. Hence $\operatorname{Im} f \subseteq \operatorname{Im} p$ has the form

$$
a=\left(\begin{array}{ccccc}
0 & * & 0 & \ldots & 0 \\
0 & 0 & * & \ldots & 0 \\
\vdots & \vdots & \ddots & \ddots & \vdots \\
0 & 0 & 0 & \ddots & * \\
* & 0 & \ldots & 0 & 0
\end{array}\right) .
$$

Each of the starred entries of $a$ is a polynomial with respect to $t_{k, i}$ and each of them takes nonzero values because $e_{k, k+1}$ belongs to the image of $f$ for any $1 \leq k \leq n-1$ and also $e_{n, 1} \in \operatorname{Im}(f)$. Therefore for generic $t_{k, \ell}$ each of the starred entries is nonzero, so the minimal polynomial of $a$ is $\lambda^{n}-\alpha$ for some $\alpha$, implying $a$ has eigenvalues $\left\{c, c \varepsilon, \ldots, c \varepsilon^{n-1}\right\}$ where $c$ is the $n$-th root of the determinant $\alpha$.

Remark 4. The variety of $n \times n$ matrices with a given set of $n$ distinct eigenvalues has dimension $n^{2}-n$.

Remark 5. Assume for some matrix units $a_{i}$ that $p\left(a_{1}, \ldots, a_{m}\right)$ is diagonal. Then $f$ as constructed in (2) in the proof of Theorem 3 is diagonal. If the dimension of the image of $\operatorname{Im} f$ is $\delta$, then each value of $f$ has some set of eigenvalues of $p$ and therefore by Remark 4, Im $p$ has dimension at least $n^{2}-n+\delta$.

As a special case, if $p$ is power-central then $\operatorname{Im} p$ has dimension $n^{2}-n+1$.

Theorem 4. If a multilinear polynomial $p$ is $\nu$-central on $M_{n}(K)$, where Char $(K)$ does not divide $n$, then then $\nu=n$.

Proof. Passing to the algebraic closure, we apply Theorem 3 to Lemma 6 
Corollary 1. If a multilinear polynomial $p$ evaluated on $n \times n$ matrices is $\nu$-central then $\nu=n$.

Proof. The matrices in the image can only have $\nu$ distinct eigenvalues, contradicting the theorem unless $\nu=n$.

Let us introduce the tool of "harmonic bases" of the space of diagonal matrices.

Remark 6. Assume that $K$ has the form $F[\varepsilon]$, where $\varepsilon$ is a primitive $n$-th root of 1 . Let - denote the automorphism of $K$ sending $\varepsilon \mapsto \varepsilon^{-1}$. Take the base of the diagonal matrices $e_{k}=\operatorname{Diag}\left\{1, \varepsilon^{k}, \varepsilon^{2 k}, \ldots, \varepsilon^{(n-1) k}\right\}, 0 \leq k \leq n-1$. Assume that there exist matrix units $a_{1}, \ldots, a_{m}$ such that $p\left(a_{1}, \ldots, a_{m}\right)=\operatorname{Diag}\left\{c_{0}, \ldots, c_{n-1}\right\}$. This can be written as a linear combination of the $e_{k}$. Assume also that the image of $p$ is at most $\left(n^{2}-n+2\right)$-dimensional. By Remark 5 , the image of $f$ constructed in the proof of Theorem 3 is at most 2-dimensional and thus is a linear space. If $p\left(a_{1}, \ldots, a_{m}\right)=h_{0} e_{0}+h_{1} e_{1}+\cdots+h_{n-1} e_{n-1}$ with $h_{k} \neq 0$, then $e_{k}$ belongs to the linear span of $\operatorname{Im} f$. Hence there are at most two nonzero coefficients, say, $h_{k}$ and $h_{l}$ with all of the others zero. We can consider the scalar product

$$
\left\langle\left\{\alpha_{1}, \ldots, \alpha_{n}\right\}\left\{\beta_{1}, \ldots, \beta_{n}\right\}\right\rangle=\sum_{i=1}^{n} \alpha_{i} \overline{\beta_{i}} .
$$

We compute $\left.\left\langle\left\{c_{0}, \ldots, c_{n-1}\right\}, e_{s}\right\}\right\rangle$ in two ways, first as $n q_{s}$ and then as

$$
c_{0}+c_{1} \varepsilon^{-s}+\cdots+c_{n-1} \varepsilon^{-(n-1) s}
$$

since $\overline{\varepsilon^{l}}=\varepsilon^{-l}$.

Theorem 5. Assume $n \geq 4$. For Char $(K)$ arbitrary, there are no multilinear power central polynomials. Any multilinear polynomial is either PI, or central or its image is at least $\left(n^{2}-n+2\right)$-dimensional.

Proof. Assume that $p$ is an $n$-central polynomial. Then there exist a set of matrix units $a_{i}$ such that $p\left(a_{1}, \ldots, a_{m}\right)=\operatorname{Diag}\left\{c_{0}, \ldots, c_{n-1}\right\}$ is diagonal but not scalar. Then the image of $f$ as constructed in (2) must be 1-dimensional, implying $\operatorname{Diag}\left\{c_{0}, \ldots, c_{n-1}\right\}$ is proportional to $\operatorname{Diag}\left\{c_{i}, c_{i+1}, \ldots, c_{n-1}, c_{0}, \ldots, c_{i-1}\right\}$ for any $i$. If at least one of the $c_{i}$ were zero, then each $c_{i}$ would be zero and therefore this matrix is zero (in particular it is scalar), a contradiction. Thus, without loss of generality $c_{0} \neq c_{1}$ are nonzero. Therefore there also exist a set of matrix units $\tilde{a}_{i}$ such that $p\left(\tilde{a}_{1}, \ldots, \tilde{a}_{m}\right)=\operatorname{Diag}\left\{c_{1}, c_{0}, c_{2}, \ldots, c_{n-1}\right\}$. We can construct the mappings $f$ and $\tilde{f}$ as before, and their images cannot be both 1-dimensional since otherwise

$$
\tau=\frac{c_{1}}{c_{0}}=\frac{c_{2}}{c_{1}}=\frac{c_{3}}{c_{2}}=\cdots=\frac{c_{n}}{c_{n-1}}=\frac{c_{0}}{c_{n}}
$$

and also $\tilde{\tau}=\frac{c_{0}}{c_{1}}=\frac{c_{2}}{c_{0}}=\frac{c_{3}}{c_{2}}=\cdots$. Hence

$$
\tau^{2}=\frac{c_{2}}{c_{1}} \cdot \frac{c_{1}}{c_{0}}=\frac{c_{2}}{c_{0}}=\tilde{\tau}=\frac{c_{3}}{c_{2}}=\tau .
$$

Thus $\tau \in\{0,1\}$. If $\tau=1$ then $p\left(a_{1}, \ldots, a_{m}\right)$ is scalar, a contradiction. If $\tau=0$ then $c_{1}=0$, a contradiction. We conclude that $\operatorname{Im} f$ is least 2-dimensional and $\operatorname{Im} p$ is at least $\left(n^{2}-n+2\right)$-dimensional. In particular $p$ is not power central.

Let us improve the estimates of the dimension of $\operatorname{Im} p$, for $n \geq 5$. 
Theorem 6. Suppose the ground field $K$ is as in Remark 6 . Let $p$ be any multilinear polynomial evaluated on $n \times n$ matrices which is not PI or central. Assume that the characteristic of $K$ does not divide $n$. If $n \geq 5$, then the image of $p$ is at least $\left(n^{2}-n+3\right)$-dimensional.

Proof. The polynomial $p$ is neither PI nor central thus there exist matrix units $a_{1}, \ldots, a_{m}$ such that $p\left(a_{1}, \ldots, a_{m}\right)=\operatorname{Diag}\left\{c_{0}, \ldots, c_{n-1}\right\}$ is diagonal but not scalar. Assume that the image of $p$ is at most $\left(n^{2}-n+2\right)$-dimensional. As we showed in Remark 6, the matrix $\operatorname{Diag}\left\{c_{0}, \ldots, c_{n-1}\right\}$ can be written as $\alpha e_{k}+\beta e_{l}$, which is not scalar. Without loss of generality we may assume that $c_{0} \neq c_{1}$ (because there exists $r$ such that $c_{r} \neq c_{r+1}$ ), and we now consider the matrix

$$
\operatorname{Diag}\left\{c_{r}, c_{r+1}, \ldots, c_{n-1} c_{0}, c_{1}, \ldots, c_{r-1}\right\}
$$

instead of our. with its different coefficients $\tilde{\alpha}=\varepsilon^{r k} \alpha$ and $\tilde{\beta}=\varepsilon^{r l} \beta$ ).

We define the matrices $q_{k}:=\operatorname{Diag}\left\{\varepsilon^{k}, 1, \varepsilon^{2 k}, \varepsilon^{3 k}, \ldots, \varepsilon^{(n-1) k}\right\}$. Switching the indices 1 and 2, we obtain matrix units $\tilde{a}_{i}$ such that

$$
p\left(\tilde{a}_{1}, \ldots, \tilde{a}_{m}\right)=\operatorname{Diag}\left\{c_{1}, c_{0}, c_{2}, c_{3}, \ldots, c_{n-1}\right\}=\alpha q_{k}+\beta q_{l} .
$$

By Remark [6, $\alpha q_{k}+\beta q_{l}$ also can be written as a linear combination of two elements of the base $e_{s}$ (say, $\tilde{\alpha} e_{\tilde{k}}+\tilde{\beta} e_{\tilde{l}}$ ). Note that

$$
\left\langle q_{k}, e_{s}\right\rangle=\varepsilon^{k}+\varepsilon^{-s}-1-\varepsilon^{k-s}=\left(\varepsilon^{k}-1\right)\left(1-\varepsilon^{-s}\right)+\left\langle e_{k}, e_{s}\right\rangle .
$$

Thus, if $k \neq s$, then

$$
\left\langle q_{k}, e_{s}\right\rangle=\varepsilon^{k}+\varepsilon^{-s}-1-\varepsilon^{k-s}=\left(\varepsilon^{k}-1\right)\left(1-\varepsilon^{-s}\right)
$$

since $\left\langle e_{k}, e_{s}\right\rangle=0$, and if $k=s$ then

$$
\left\langle q_{k}, e_{s}\right\rangle=\left(\varepsilon^{k}-1\right)\left(1-\varepsilon^{-s}\right)+n .
$$

Hence, if $s \notin\{k, l\}$ then

$$
\left\langle\alpha q_{k}+\beta q_{l}, e_{s}\right\rangle=\left(1-\varepsilon^{-s}\right)\left(\alpha\left(\varepsilon^{k}-1\right)+\beta\left(\varepsilon^{l}-1\right)\right) .
$$

We denote $\delta=\alpha\left(\varepsilon^{k}-1\right)+\beta\left(\varepsilon^{l}-1\right)$. Recall that $c_{1} \neq c_{0}$, and thus $\delta \neq 0$. Therefore either $s=\tilde{k}$, or else $s=\tilde{l}$, or $\left\langle\alpha q_{k}+\beta q_{l}, e_{s}\right\rangle=0$ (and thus $s$ is either $k$, or $l$, or $1-\varepsilon^{-s}=0$ (and thus $s=0$ ) - only five possibilities. But for $n \geq 6$ there are at least three nonzero coefficients, a contradiction.

Thus we may assume that $n=5$. We have exactly five possibilities for $s$, which therefore must be distinct. Therefore the $k$-th and $l$-th coefficients of $\alpha q_{k}+\beta q_{l}$ will be zero, i.e.,

$$
\left(1-\varepsilon^{-k}\right) \delta+5 \alpha=\left(1-\varepsilon^{-l}\right) \delta+5 \beta=0,
$$

where $\delta=\alpha\left(\varepsilon^{k}-1\right)+\beta\left(\varepsilon^{l}-1\right)$. In particular

$$
\frac{\alpha}{\beta}=\frac{1-\varepsilon^{-k}}{1-\varepsilon^{-l}} .
$$

Now let us take matrix units $a_{i}^{\prime}$ such that $p\left(a_{1}^{\prime}, \ldots, a_{m}^{\prime}\right)=\operatorname{Diag}\left\{c_{2}, c_{1}, c_{0}, c_{3}, c_{4}\right\}$. Then $\alpha r_{k}+\beta r_{l}$ can also be written as a linear combination of two of the $e_{s}$, where $r_{k}=\operatorname{Diag}\left\{\varepsilon^{2 k}, \varepsilon^{k}, 1, \varepsilon^{3 k}, \varepsilon^{4 k}\right\}$. If $k \neq s$, then

$$
\left\langle r_{k}, e_{s}\right\rangle=\varepsilon^{2 k}+\varepsilon^{-2 s}-1-\varepsilon^{2 k-2 s}=\left(\varepsilon^{2 k}-1\right)\left(1-\varepsilon^{-2 s}\right) .
$$


We perform the same calculations as before, and obtain

$$
\frac{\alpha}{\beta}=\frac{1-\varepsilon^{-2 k}}{1-\varepsilon^{-2 l}} .
$$

Hence

$$
\frac{1-\varepsilon^{-k}}{1-\varepsilon^{-l}}=\frac{1-\varepsilon^{-2 k}}{1-\varepsilon^{-2 l}},
$$

implying

$$
\frac{1+\varepsilon^{-k}}{1+\varepsilon^{-l}}=1
$$

and hence $k=l$, a contradiction.

Theorem 7. Let $p$ be any multilinear polynomial evaluated on $4 \times 4$ matrices, which is neither PI nor central. Assume that Char $K \neq 2$. Then $\operatorname{dim} \operatorname{Im} p \geq 14$, equality holding only if the following conditions are satisfied:

- For any matrix units $a_{i}$, if $p\left(a_{1}, \ldots, a_{m}\right)$ is diagonal then it has eigenvalues $(c, c,-c,-c)$ for some $c$.

- Any value of $p$ has eigenvalues $\left(\lambda_{1}, \lambda_{2},-\lambda_{1},-\lambda_{2}\right)$.

Proof. First note that $4^{2}-4+2=14$, so $\operatorname{dim} \operatorname{Im} p \geq 14$. Assume that $p$ is a multilinear polynomial evaluated on $4 \times 4$ matrices with 14-dimensional image. Let $a_{1}, \ldots, a_{m}$ be any matrix units such that $p\left(a_{1}, \ldots, a_{m}\right)$ is diagonal but not scalar. Let $p\left(a_{1}, \ldots, a_{m}\right)=\operatorname{Diag}\left\{c_{0}, c_{1}, c_{2}, c_{3}\right\}$ and $c_{0} \neq c_{1}$. We use the same notation as in the proof of Theorem 6. Recall that $e_{k}=\operatorname{Diag}\left\{1, i^{k}, i^{2 k}, i^{3 k}\right\}$ and $q_{k}=\operatorname{Diag}\left\{i^{k}, 1, i^{2 k}, i^{3 k}\right\}$. As in the proof of Theorem $\left[\alpha q_{k}+\beta q_{l}, e_{s}\right\rangle=\delta\left(1-i^{-s}\right)$ if $s \notin\{k, l\}$, or $\delta\left(1-i^{-s}\right)+4 \alpha$ if $s=k$ and $\delta\left(1-i^{-s}\right)+4 \beta$ if $s=l$. Therefore $k$ and $l$ are nonzero (for otherwise we have two nonzero possibilities for $s \notin\{k, l\}$ and one other nonzero coefficient would be zero: $\left\langle\alpha q_{k}+\beta q_{l}, e_{0}\right\rangle=4 \alpha$ (if we assume $k=0$ without loss of generality). Therefore $p\left(a_{1}, \ldots, a_{m}\right)$ belongs to the linear span $\left\langle e_{1}, e_{2}, e_{3}\right\rangle$. Hence we have three options:

- $p\left(a_{1}, \ldots, a_{m}\right)=\alpha e_{1}+\beta e_{2}$,

- $p\left(a_{1}, \ldots, a_{m}\right)=\alpha e_{1}+\beta e_{3}$,

- $p\left(a_{1}, \ldots, a_{m}\right)=\alpha e_{3}+\beta e_{2}$.

We will not treat the last case since its calculations are as in the first case. Let us consider the first case $p\left(a_{1}, \ldots, a_{m}\right)=\alpha e_{1}+\beta e_{2}$. Therefore $p\left(\tilde{a}_{1}, \ldots, \tilde{a}_{m}\right)=$ $\alpha q_{1}+\beta q_{2}$ which can be written explicitly as

$$
\left(\frac{1}{2} \alpha-\frac{1+i}{2} \beta\right) e_{1}+\frac{i-1}{2} \alpha e_{2}+\left(\frac{i}{2} \alpha+\frac{i-1}{2} \beta\right) e_{3},
$$

thus $\alpha \in\{0,(1+i) \beta,-(1+i) \beta\}$. If $\alpha=(1+i) \beta$ then $p\left(a_{1}, \ldots, a_{m}\right)=\beta \operatorname{Diag}\{i-$ $2, i+2,-i,-i\}$. If $\alpha=-(1+i) \beta$ then $p\left(a_{1}, \ldots, a_{m}\right)=\beta \operatorname{Diag}\{-i,-i, i+2, i-2\}$. In both cases $\alpha= \pm(1+i) \beta$, so there are matrix units $\tilde{a}_{i}$ such that $p\left(\tilde{a}_{1}, \ldots, \tilde{a}_{m}\right)=$ $\operatorname{Diag}\{-i, i+2,-i, i-2\}$ which can be written explicitly as $-i e_{1}-i e_{2}+i e_{3}$, and we have three nonzero coefficients. We conclude that the image is at least 15dimensional. If $\alpha=0$, then the value $p\left(a_{1}, \ldots, a_{m}\right)$ has eigenvalues $(c, c,-c,-c)$ as in the conditions of the Theorem. 
Assume now $p\left(a_{1}, \ldots, a_{m}\right)=\alpha e_{1}+\beta e_{3}$. Therefore $p\left(a_{1}, \ldots, a_{m}\right)=\operatorname{Diag}\{x, y,-x,-y\}$. Then we consider $p\left(\tilde{a}_{1}, \ldots, \tilde{a}_{m}\right)=\operatorname{Diag}\{x,-x, y,-y\}$ which can be written explicitely as

$$
\frac{(x-y)(1+i)}{4} e_{1}+\frac{x+y}{2} e_{2}+\frac{(x-y)(1-i)}{4} e_{3},
$$

therefore $x= \pm y$ and once again we have a matrix from the conditions of the theorem.

Therefore there is a set of matrix units $a_{i}$ with $p\left(a_{1}, \ldots, a_{m}\right)=\operatorname{Diag}\{c, c,-c,-c\}$. Now we construct the mapping $f$ and the image will be 2-dimensional if and only if it is the set $\operatorname{Diag}\left\{\lambda_{1}, \lambda_{2},-\lambda_{1},-\lambda_{2}\right\}$. Therefore $\operatorname{Im} p$ contains all the matrices with such eigenvalues, which is a 14 -dimensional variety. Hence, if $\operatorname{dim} \operatorname{Im} p=14$, then $\operatorname{Im} p$ is exactly this variety.

\section{OPEN PROBLEMS}

The following questions remain from this paper:

Problem 1. Does there actually exist a multilinear 3-central polynomial for $n=3$ ? We can prove that these do not exist (although homogenous ones do exist).

Problem 2. Does there exist an n-central polynomial (not multilinear) for $n>3$ prime? This would answer the celebrated cyclicity question for division algebras of prime degree.

\section{REFERENCES}

[BMR1] Belov, A.; Malev, S.; Rowen, L. The images of non-commutative polynomials evaluated on $2 \times 2$ matrices, Proc. Amer. Math. Soc 140 (2012), 465-478.

[BMR2] Belov, A.; Malev, S.; Rowen, L. The images of multilinear polynomials evaluated on $3 \times 3$ matrices, preprint (2013).

[F1] Formanek, E. Central polynomials for matrix rings, J. Algebra 23 (1972), 129-132.

[F2] Formanek, E. The polynomial identities and invariants of $n \times n$ matrices, CBMS Regional Conf. Series in Math. 78 (1991), 129-132.

[Ra1] Razmyslov, Yu. On a problem of Kaplansky (Russian), Izv. Akad. Nauk SSSR., Ser. Mat. 37 (1973), 483-501. (Translation: Math USSR., Izv. 7 (1973), 479-496.)

[Row] Rowen, L. Polynomial identities in ring theory, Academic press, Pure and Applied Math., New York (1980).

[S] Saltman, D. On p-power central polynomials, Proc. Amer. MAth. Soc. 78 (1980),.

Department of mathematics, Bar Ilan University, Ramat Gan, Israel

E-mail address: beloval@math.biu.ac.il

E-mail address: malevs@math.biu.ac.il

E-mail address: rowen@math.biu.ac.il 